\title{
Development of an Amine-based System for Combined Carbon Dioxide, Humidity, and Trace Contaminant Control
}

\author{
Tim Nalette and Julie Reiss, Hamilton Sundstrand \\ Tom Filburn, University of Hartford \\ Thomas Seery and Bob Weiss, University of Connecticut \\ Fred Smith and Jay Perry, NASA
}

\begin{abstract}
A number of amine-based carbon dioxide $\left(\mathrm{CO}_{2}\right)$ removal systems have been developed for atmosphere revitalization in closed loop life support systems. Most recently, Hamilton Sundstrand developed an amine-based sorbent, designated SA9T, possessing approximately 2 -fold greater capacity compared to previous formulations. This new formulation has demonstrated applicability for controlling $\mathrm{CO}_{2}$ levels within vehicles and habitats as well as during extravehicular activity (EVA). System volume is competitive with existing technologies. Further enhancements in system performance can be realized by incorporating humidity and trace contaminant control functions within an amine-based atmosphere revitalization system. A 3-year effort to develop prototype hardware capable of removing $\mathrm{CO}_{2}, \mathrm{H}_{2} \mathrm{O}$, and trace contaminants from a cabin atmosphere has been initiated. Progress pertaining to defining system requirements and identifying alternative amine formulations and substrates is presented.
\end{abstract}

\section{Introduction}

Long term Space Exploration missions require regenerable systems to minimize resupply logistics. Current methods of $\mathrm{CO}_{2}$ removal for aerospace life support systems include non-regenerable lithium hydroxide $(\mathrm{LiOH})$, and regenerable systems that employ silver oxide, solid amines or molecular sieves.[References?] In 2004 a 3-year rapid technology development project was initiated with the objective of delivering a prototype regenerable, amine-based system that combines $\mathrm{CO}_{2}$, humidity, and trace contaminant (TC) control within a single system. The project team includes members from industry, academia, and NASA.

This project will result in the development, design, fabrication, and test of a full-scale prototype system capable of combined $\mathrm{CO}_{2}$, humidity, and TC control. At the project's conclusion, system-level testing of the prototype will demonstrate a Technology Readiness Level (TRL) 6, with subsequent delivery of the prototype hardware to NASA. This paper presents background and the project's status after the first year's effort.

\section{Background}

Research by Hamilton Sundstrand spanning several decades has demonstrated the reliability of solid amines for scrubbing $\mathrm{CO}_{2}$ from air. These sorbents have also shown their ability to remove water vapor as well as some trace contaminants from gas streams. This project combines advances in amine chemistry with alternative, high pore volume 
carbon substrate materials and evaluates their potentially enhanced TC control capabilities. Combining higher amine loadings with carbon-based supports has the potential for providing $\mathrm{CO}_{2}$, humidity, and TC control capability with a single regenerable sorbent.

In addition to reducing re-supply logistics compared to non-regenerable technologies, integrating the humidity control function into the amine eliminates the need for condensing heat exchangers and potentially allows higher radiator temperature and smaller radiator surface area. The amine based sorbents operate as a pressure-swing absorption (PSA) process, requiring much less power than other regenerable systems that require high temperatures for enhancing regeneration kinetics. Additionally, recent developments in amine chemistry allow the sorbent to operate under low humidity conditions so that a moisture recovery system can be integral to future systems as need dictates.

The first 12-15 months of the 3-year project includes research that will evaluate methods for enhancing sorbent performance and lowering manufacturing costs of the developmental hardware. The remainder of the project focuses on the design, development, fabrication, and testing of full-scale prototype hardware.

New amine formulations developed by Hamilton Sundstrand have demonstrated approximate 2 -fold $\mathrm{CO}_{2}$ capacity increase compared to previous formulations used to demonstrate regenerative $\mathrm{CO}_{2}$ removal on board the Space Shuttle orbiter (1). The higher capacities now allow conceptual application to extravehicular activity (EVA) systems that can be packaged within volume constraints equivalent to existing metal oxide (Metox)-based equipment. Also, fully redundant vehicle systems that have approximately the same volume as previous, non-redundant amine-based systems are possible. A typical breakthrough curve comparing the new amine formulation, designated SA9T, to the previously flight-certified regenerable carbon dioxider removal system (RCRS) sorbent, designated $\mathrm{HSC}^{+}$, is shown in Figure 1. 


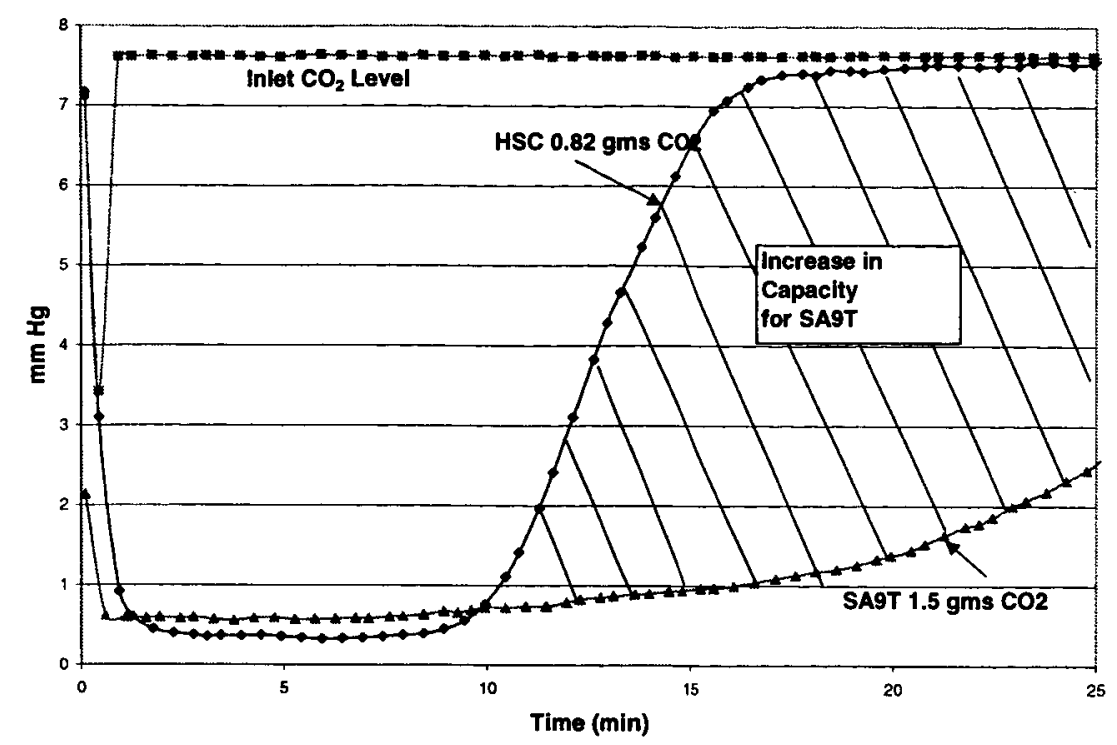

Figure 1 Increased Capacity of SA9T Formulation

In addition to its high capacity for cyclically removing $\mathrm{CO}_{2}$ from a gas stream, SA9T has demonstrated high capacity for removing water vapor. Figure 2 shows sub-scale test results of SA9T's $\mathrm{H}_{2} \mathrm{O}$ capacity when operated at dew points close to those expected during a typical EVA. This capacity represents approximately $2 / 3$ of the moisture removal required without any enhancement of the baseline SA9T formulation.

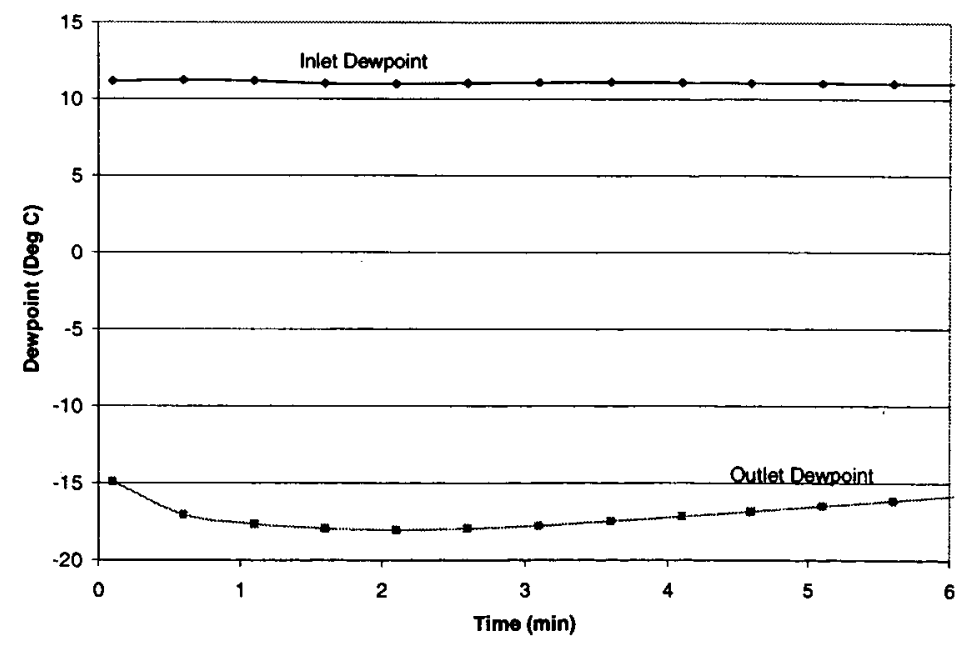

Figure 2 SA9T Cyclic Capacity for Moisture Removal

In addition to adsorption of $\mathrm{CO}_{2}$ and $\mathrm{H}_{2} \mathrm{O}$, previous amine formulations have demonstrated capacity for removing trace contaminants. Trace contaminant removal by amine-based sorbents has been shown in many cases to be reversible.(2) High water retention and sorption capacity also serve to enhance trace contaminant removal. Based on the water solubility of the contaminant challenges, approximately $25 \mathrm{cc} /$ day of water is required to remove all water-soluble contaminants from the present Extravehicular 
Mobility Unit (EMU) vent loop. This includes metabolically produced ammonia in addition to alcohols, ketones (organic and inorganic), and nitrogen- and sulfur-containing organic compounds.

Since the SA9T sorbent has a much higher density of immobilized amine compared to the $\mathrm{HSC}^{+}$sorbent, it is possible that the capacity for trace contaminants may increase. Further, it is likely that a carbon-based substrate material may further enhance the sorbent's capacity for trace contaminants.

Implementing the solid amine technology requires some method for managing thermal loads because adsorption is exothermic. To maximize $\mathrm{CO}_{2}$ loading, the sorbent must be cooled to remove the heat of adsorption. Conversely, the desorbing bed must be heated efficiently remove the $\mathrm{CO}_{2}$. This is accomplished by building a series of adjacent sorbent sections that alternate between adsorption and desorption zones. Each section is fabricated with integral, thermally conductive metallic foam elements to enhance the heat transfer between adjacent sections. A cross section showing the amine sorbent loaded in a section of metallic foam is shown in Figure 3.

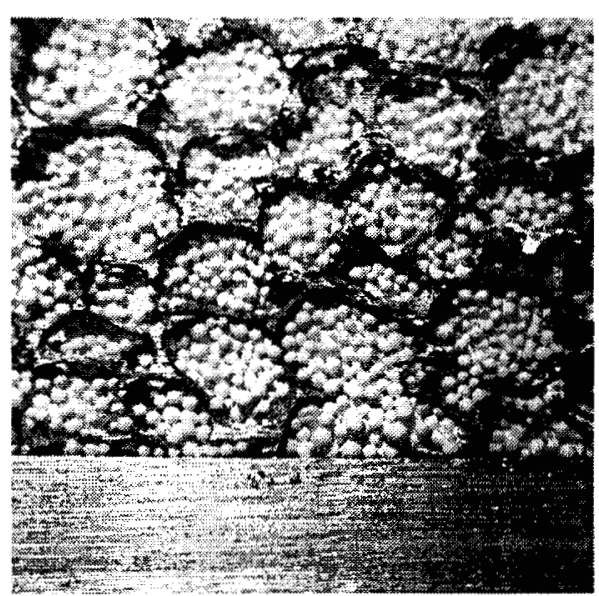

Figure 3 Solid Amine Sorbent in Thermally Conductive Metal Foam

To use the amine sorbent technology in a PSA system, the sorbent sections are sized based on thermal considerations, and then optimized in terms of weight and volume based on the specific application. Studies conducted for EVA and vehicle applications indicate bed volumes may range from approximately $0.13 \mathrm{ft}^{3}$ to $0.35 \mathrm{ft}^{3}$, respectively. Corresponding adsorption/desorption half-cycles may range from approximately 6 minutes to 30 minutes. For PSA operation, the metallic foam-loaded sorbent sections are coupled to a valve assembly that periodically exposures alternating bed sections to space vacuum to desorb the $\mathrm{CO}_{2}, \mathrm{H}_{2} \mathrm{O}$, and trace contaminants. This is shown conceptually by Figure 4. 


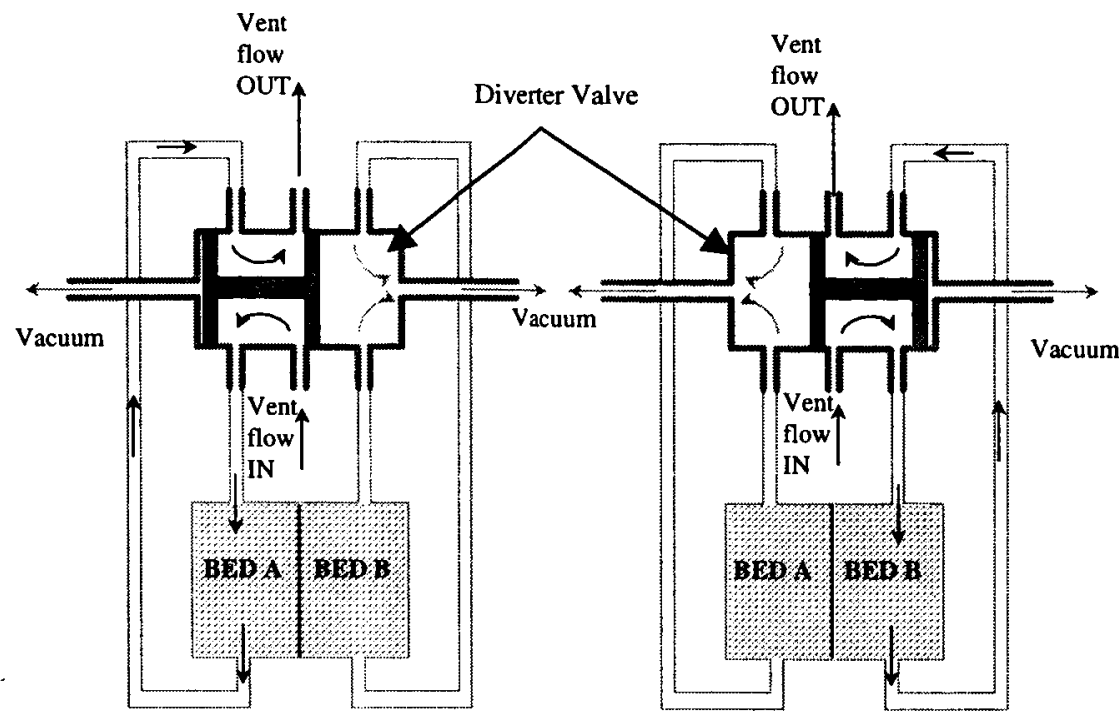

Figure 4. Two-Bed Solid Amine-Based $\mathrm{CO}_{2}$, Humidity and Contaminant Control Schematic

Basic implementation of the solid amine concept uses thermally-linked beds to contain the amine sorbent coupled with periodic exposure to space vacuum to effect regeneration. The exothermic heat of reaction of the adsorbing bed is transferred to the adjacent regenerating bed, which, when combined with exposure to vacuum, provides the energy necessary for desorption. A diverter valve directs air flow to one bed while simultaneously exposing the other bed to space vacuum. After a predetermined interval, the valve position changes to expose the regenerated bed to air flow and the saturated bed to space vacuum.

Figures 5 and 6 show typical results obtained from a subscale, multiple-bed test article. Figure 5 is representative of a fully-redundant system sized to fit in the same volume occupied by the RCRS hardware used during Extended Duration Orbiter missions in the 1990s. Figure 6 represents a nominal and maximum metabolic rate typical of EVA applications.

Julie -Add cyclic data showing typical operation at anticipated vehicle and emu conditions - figures $5 \& 6$

\section{Rapid Technology Development Project}

The rapid technology development project is a 3-year effort. The initial phase consists of approximately 12-15 months of research designed to increase sorbent performance and simplify the canister design. The later part of the program focuses on designing, fabricating, assembling, and testing a full-scale functional prototype. The project team consists of experts from academia, industry, and the NASA. Hamilton Sundstrand is responsible for the overall project as well as a portion of the basic research associated 
with the enhancing the sorbent, improving the system-level design, and building the fullscale prototype. NASA assists with definiting requirements and evaluating the sorbent for trace contaminant control capacity. University partners, including the University of Hartford and the University of Connecticut, will fabricate specialty amine based sorbents that maximize secondary amine content, while industrial partners will investigate higher pore volume supports that will increase the loading density of immobilized amine, thereby increasing sorbent performance.

The research phase will evaluate potential enhancements to the amine sorbent that will include optimized chemistries based on the SA9T formulation and alternative substrates to enhance $\mathrm{CO}_{2}$, humidity, and trace contaminant capacities. The optimized chemistries will focus on maximizing the quantity of secondary amine in the sorbent. Two substrate types will be evaluated-substrates having high pore volumes that permit higher amine loadings and carbon-based substrates that potentially aid in trace contaminant control. Depending on the application, the system may be sized for a vehicle, for advanced EVA, or as a common system to accommodate both.

\section{Current Status}

During the project's first year, Hamilton Sundstrand has identified candidate alternative substrates to the baseline poly methyl methacrylate (PMMA). The substrates identified for further evaluation are listed in Table 1 and have been selected for their potential benefits for increasing $\mathrm{CO}_{2}$ loading and trace contaminant adsorption potential.

\section{Julie-Table 1 - Candidate Sorbent Supports}

The screening process first determines the sorbent's $\mathrm{CO}_{2}$ adsorption capacity and kinetic behavior. If it is determined to be competitive with the baseline formulation, then it is tested further to determine its performance for humidity and TC control. To date, the substrates indicated in bold in Table 1 have been used to formulate amine sorbents and to conduct screening tests for comparison to with the baseline SA9T sorbent. Results of the initial screening tests are shown in Figure 6. The data indicate that the alternative sorbents tested thus far do not perform as well as the baseline SA9T. Therefore they are not considered viable alternatives.

\section{Julie - Figure 6 - comparative test results of initial screening tests}

Literature searches on unique synthesis chemistries that may be possible alternatives to amines have been completed by the University of Hartford and the University of Connecticut.

The University of Hartford is investigating the synthesis and adsorption properties of large amine dendrimer molecules and other large chain molecules containing amine functionality (e.g Polyvinylamine PVA). The goal of this effort is to produce materials 
possessing a high degree of secondary amine functionality and sufficient molecular weight to produce a solid material. These larger molecules will obviate the need for a support structure, and theoretically can produce a sorbent with high specific capacities compared to current immobilized configurations. In addition to the amine synthesis effort, the University of Hartford has designed and fabricated an adsorption test rig for testing the new amine formulations. This test rig will also be used to test sorbents developed by the University of Connecticut.

An alternative synthesis route is being investigated by The University of Connecticut in which the amine functionality is inherent in the polymer support, analogous in many ways to a weak base ion exchange resin. The goal is to maximize the number of secondary amine groups in the basic structure. This research will attempt to optimize the amine spacing and amine loading, as well as investigate the oxidative resistance of the sorbents. To date a number of amines have been manufactured which will be tested using thermo-gravimetric techniques to measure the equilibrium $\mathrm{CO}_{2}$ capacity.

If any of the alternative amine formulations show promising results in the screening tests, then larger quantities (approximately $300 \mathrm{cc}$ ) will be manufactured and tested by Hamilton Sundstrand in a 2-bed, sub-scale reactor to evaluate cyclic performance.

A 2-bed, subscale reactor has been manufactured and loaded with the baseline SA9T and delivered to NASA for TC control testing. Test rig modifications are being implemented for this testing. A picture of the test hardware is shown in figure 8. The reactor is a four chamber reactor, with alternating cambers connected externally to provide a 2 -bed configuration.

\section{Julie - Figure 8 - 2 Bed Sub-scale Test Reactor}

A representative contaminant challenge has been defined based on ISS in-flight cabin air quality measurements and previous evaluation of amines for their TC control capacity. Figure 9 shows the average trace component composition.(3) [Ref. Peterson \& Perry, 2002] The total average trace contaminant concentration is $20.2 \mathrm{mg} / \mathrm{m}^{3}$. Methane typically dominates the overall trace component composition. Previous evaluations of amines for their interaction with contaminants and their TC control capacity have used methanol, ethanol, isopropanol, benzene, carbon monoxide, methane, acetone, dichloromethane, Halon 1301 (bromotrifluoromethane), trichloroethane, Freon 21 (dichlorofluoromethane), and decamethylcylcopentasiloxane.(2,4,5,6) [Ref. 2 and SAE 921349, 932293, and 951540] The most prevalent contaminants observed during flight include ethanol, acetaldehyde, dimethylbenzenes, dichloromethane, acetone, methane, carbon monoxide, hydrogen, and hexamethylcyclotrisiloxane. These compounds at 20.2 $\mathrm{mg} / \mathrm{m}^{3}$ total distributed according to Figure 9 serve as the TC challenge basis. Other compounds used during previous evaluations of amine TC control capacity. The total concentration may be increased to enhance test analytical accuracy and precision. 


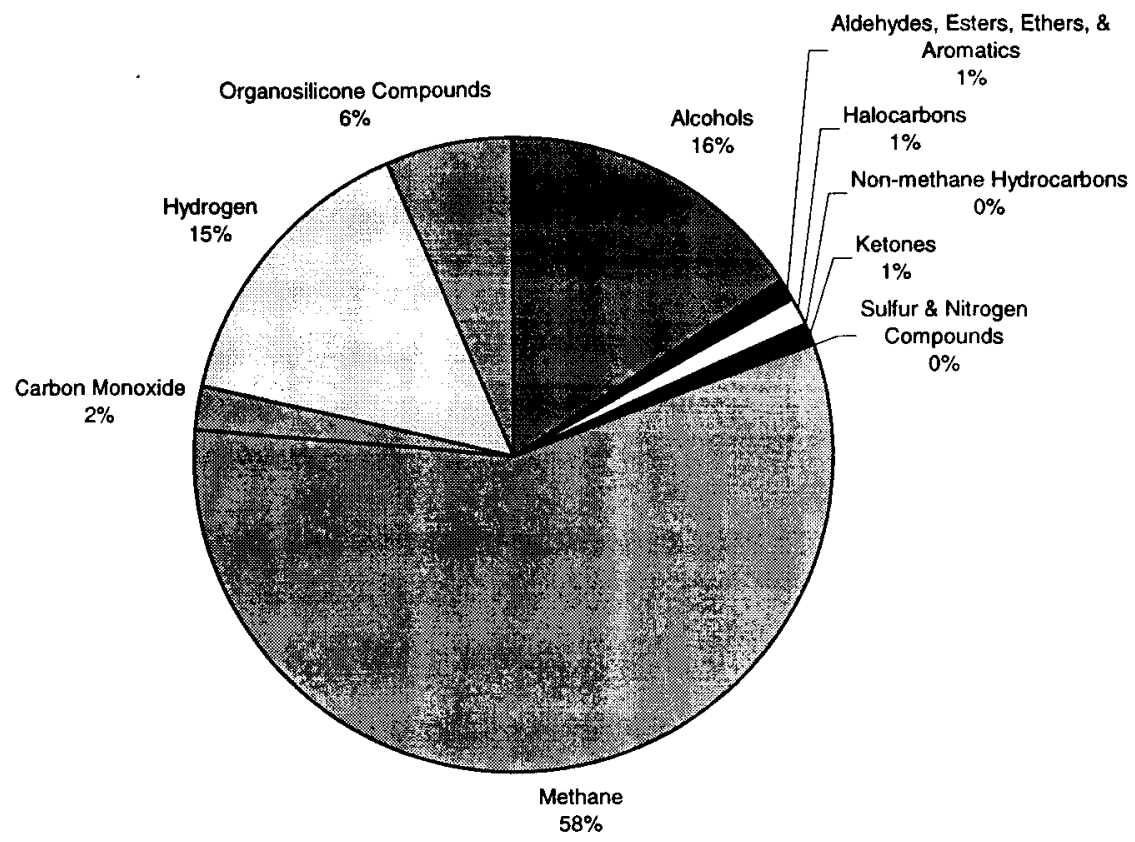

Figure 9. Average Trace Contaminant Load Composition in the ISS U.S. Lab

In February 2005, a Systems Requirement Review (SRR) was successfully completed. The SRR established the requirements for developing, manufacturing, and testing the prototype hardware. A partial listing of the pertinent requirements is given in Table 2.

\section{Julie - Table 2 - Partial listing of pertinent requirements}

\section{Future Effort}

During project's second year, sorbent development and evaluation will continue including alternative substrate testing by Hamilton Sundstrand and alternative amine development by the university partners. At the conclusion of the sorbent development phase the most promising sorbent formulation will be selected and used in the prototype hardware. TC control screening using the baseline and alternative sorbents that have shown sufficient performance in the screening tests will continue at NASA. After selecting the sorbent, preliminary design of the sorbent reactor and valve assembly will be initiated.

The project's final year will focus on fabricating, assembling, and testing of the prototype hardware followed by delivery to NASA.

\section{Summary}


The first year of a rapid technology development project for developing a system for combined $\mathrm{CO}_{2}$, humidity, and $\mathrm{TC}$ control has identified numerous, alternative amine substrates that may enhance performance compared to the baseline SA9T sorbent.

Alternative amine chemistries have been identified that have the potential for increasing sorbent capacity and synthesis procedures are being developed by university partners. Additionally, a sub-scale reactor has been manufactured and baseline TC control testing has been initiated. Finally, a SRR to define the basic design and performance requirements for the prototype hardware was conducted.

\section{References}

1) Nalette, T., Filburn, T., Graf, J.,The Design and Testing of a Fully Redundant Regenerative $\mathrm{CO}_{2}$ Removal System (RCRS) for the Shuttle Orbiter, ICES 2001-2420, July 2001.

2) Garrard, G. G., Characterization of Solid Amine HSC+ for effects of Trace Contaminants, Rockwell International Test Report S135033, September 1993.

3) Perry, J.L. and Peteson, B.V.: Cabin Air Quality Dynamics On Board the International Space Station. SAE 2003-01-2650. July 2003.

4) Johnson, S.R. and Garrard, G.G.: Regenerative Trace Contaminant Control: New Test Method for Effects on Solid Amine. SAE 921349. July 1992.

5) Kazemi, A.R. and Mitchell, S.M.: Advanced Testing and Modeling of a Modified Solid Amine Regenerative $\mathrm{CO}_{2}$ and $\mathrm{H}_{2} \mathrm{O}$ Removal System. SAE 932293. July 1993.

6) Graf, J.C. and Spexarth, G.R.: The Impact of Trace Contaminants on the Shuttle Orbiter Regenerative $\mathrm{CO}_{2}$ Removal System. SAE 951540. July 1995.

\section{Contact Information}

Tim Nalette

Hamilton Sundstrand Space Land and Sea

860-654-2403

t.nalette@hs.utc.com 